\title{
Can Fluorine-18-Fluorodeoxyglucose Positron Emission Tomography Be Used As a Useful Method to Evaluate the Treatment Response to Neoadjuvant Therapy Combined With Sorafenib and Anti-VEGF in Children Diagnosed With Metastatical Bone Sarcoma?
}

\author{
Nurdan Tacyildiz, Hikmet Gulsah Tanyildiz,, ${ }^{1, *}$ Handan Ugur Dincaslan, Gulsan Yavuz, \\ Emel Unal, ${ }^{1}$ Elgin Ozkan, ${ }^{2}$ Cigdem Soydal, ${ }^{2}$ Ozlem Kucuk, ${ }^{2}$ and Yusuf Yildiz ${ }^{3}$ \\ ${ }^{1}$ Department of Pediatric Oncology, School of Medicine, Ankara University, Ankara, Turkey \\ ${ }_{3}^{2}$ Department of Nuclear Medicine, School of Medicine, Ankara University, Ankara, Turkey \\ 3 Department of Orthopedics, School of Medicine, Ankara University, Ankara, Turkey \\ ${ }^{*}$ Corresponding author: Hikmet Gulsah Tanyildiz, Department of Pediatric Oncology, School of Medicine, Ankara University, Ankara, Turkey. Tel:+90-5058731636, Fax: +90-3123191440, \\ E-mail:g_oktay4910@yahoo.com \\ Received 2015 September 5; Revised 2015 November 6; Accepted 2015 December 1.
}

\begin{abstract}
Background:The prognosis is still poor for patients with a metastatic bone tumor and new treatment approaches (anti-VEGFand tyrosine kinase inhibitors vs) are therefore needed.

Objectives: The aim of our study was to evaluate how the primary and metastatic lesions of our patients with a bone tumor were affected by these treatments and to determine the importance of the 18F-FDG PET method.

Patients and Methods: Twenty metastatic bone tumor cases were included. Sorafenib and anti-VEGF were added to the standard treatment in cases with widespread metastatic disease at diagnosis or after neoadjuvant chemotherapy showing less than $90 \%$ tumor necrosis in the surgical sample. Positron emission tomography (PET) imaging was performed at diagnosis, the preoperative period following neoadjuvant chemotherapy, during postoperative follow-up, and when treatment was discontinued.

Results: The primary treatment region median SUVmax level decreased from 7.35 to 2.5 in the living patients $(n=16)$ while there was no significant decrease in the patients who succumbed to the disease $(\mathrm{P}<0.001)$. Comparison of the pre- and post-treatment metastasis region median SUVmax levels in patients with metastatic involvement showed a decrease from 2.1 to 0 in the surviving patients but only from 4.8 to 3.2 in the deceased patients $(\mathrm{P}<0.01)$. Survival results indicated that $28.6 \%$ of the patients receiving classical treatment only died while all the patients receiving additional sorafenib and anti-VEGF survived.

Conclusions: 18F-PET may be a useful technique before and during the follow-up of neoadjuvant treatment in pediatric metastatic bone tumor patients. The addition of sorafenib and anti-VEGF to classical treatment has a favorable contribution to the response and therefore the survival duration.
\end{abstract}

Keywords: Bone Tumors, Sorafenib, Anti-VEGF, PET

\section{Background}

Imaging methods are important in the staging and follow-up of the treatment response in malignant bone tumor cases. The PET imaging method is especially useful in the differentiation of benign and malignant bone tumors and the evaluation of the histopathological response of the tumor to treatment (1-3). 18F-fluorodeoxyglucose enhancement has been shown to have a positive correlation with the presence of malignant bone tumors, especially in adult patients, and has also been found to be useful in the followup of pulmonary metastatic nodules. FDG PET is beneficial in detecting distant metastases when compared with conventional methods. 18F-FDG enhancement is also seen in pediatric bone tumors and it is said that the technique can be used to monitor the response to treatment (4-6). Al- though still controversial and uncertain, it is clear that PET could be a useful method for clinical practice.

Angiogenesis is required for tumor growth and metastasis. Vascular endothelial growth factor (VEGF) and its receptors (VEGFR-1 and VEGFR-2) and platelet-derived growth factor and its receptors play a major role in tumor vascularization. Bevacizumab is a monoclonal antibody binding all forms of VEGF with high affinity and is used for the treatment of solid refractory tumors in adults. A phase I study on pediatric refractory solid tumors has also reported stable disease with no drug-related toxicity (with 5,10,15 mg/kg every 2 weeks) (7).

Sorafenib is an oral multikinase inhibitor (Raf-1, BRAF, FLT-3, C-kit, VEGFR-2,VEGFR-3 and PDGFRB) and is used in

Copyright (C) 2016, Growth \& Development Research Center. This is an open-access article distributed under the terms of the Creative Commons Attribution-NonCommercial 4.0 International License (http://creativecommons.org/licenses/by-nc/4.0/) which permits copy and redistribute the material just in noncommercial usages, provided the original work is properly cited. 
adult renal cell carcinoma and inoperable hepatocellular carcinoma. The sorafenib and anti-VEGF combination potentiates the effect of each component. When used at tolerable doses, it can increase the treatment success rate in pediatric metastatic bone tumor cases with better survival rates as found in our study $(8,9)$. There is a high rate of metastatic disease at the time of diagnosis with osteosarcoma and Ewing's sarcoma and the fact that this is the most important prognostic factor emphasizes the importance of these targeted treatment agents that increase treatment success. Adding these agents to systemic treatment may increase the success in disease control as the number of chemotherapeutics that can be used with diffuse disease and relapse is limited. 18F-PET may be a useful technique before and during the follow-up of neoadjuvant treatment in pediatric metastatic bone tumor patients.

\section{Objectives}

The aim of our study was to evaluate how the primary and metastatic lesions of our patients with a bone tumor were affected by these treatments and to determine the importance of the 18F-FDG PET method.

\section{Patients and Methods}

We included a total of 20 metastatic bone tumor cases [7 Ewing sarcoma (ES) and 13 osteosarcoma (OS) cases] diagnosed between 2010 and 2013 retrospectively. There were 10 females and 10 males with a median age at diagnosis of 14 (7 - 17) years (Table 1). Median follow up time was 18 (6 - 37 ) months. Classical treatment protocols were used for bone tumors in our clinic up to 2011. The standard treatment agents used for systemic chemotherapy are cisplatin (DDP), doxorubicin (DOX) and high-dose methotrexate (HD-MTX) together with ifosfamide and/or etoposide (EURAMOS) to prevent the development of metastatic disease in osteosarcoma besides surgical treatment. We have used the classical treatment agents of cyclophosphamide, vincristine, doxorubicin, ifosfamide and etoposide (VACD-IE) alternatively for Ewing sarcoma cases in our clinic. However, we have started adding a tyrosine kinase inhibitor and anti-VEGF (recombinant monoclonal $\operatorname{IgG}_{1}$ ) to the treatment if metastatic disease was present or the case was refractory to classical treatment with $<90 \%$ necrosis since 2011 (anti-VEGF $10 \mathrm{mg} / \mathrm{kg} /$ dose once every 2 weeks and sorafenib $200 \mathrm{mg} /$ $\mathrm{m}^{2}$ /dose for 2 doses every day). We discontinued sorafenib during main chemotherapy. After chemotherapy cycle finished, we started sorafenib again. We continued anti-VEGF and sorafenib treatment for 6 months in patients refractory to treatment or residual disease. We have also been combining PET imaging with magnetic resonance at the time of diagnosis, preoperatively following neoadjuvant treatment and in the postoperative period to evaluate the treatment response since 2010 . Six of our ES cases received classical treatment and one received classical treatment combined with anti-VEGF and sorafenib while 8 of the OS cases received classical treatment and 5 additionally received anti-VEGF and sorafenib. We showed PET images of one of representative patient diagnosed Ewing sarcoma who used combination treatment successfully (Figure $1 \mathrm{~A}$ and B, Figure $2 \mathrm{~A}$ and $\mathrm{B}$ ). SPSS program was used for statistical analysis while comparing combination treatment and classical treatment results with survival and PET SUV max values of primary and metastasis region.

\begin{tabular}{|c|c|c|c|c|c|}
\hline Name & Age & Gender & Diagnosis & Sorafenib + Anti VEGF & Final State \\
\hline M.S. & 14 & $\mathrm{M}$ & OS & + & A \\
\hline Y.A. & 10 & M & OS & + & A \\
\hline M.C.Y. & 8 & M & OS & + & A \\
\hline U.O.Y. & 15 & M & OS & + & A \\
\hline B.A. & 15 & $\mathrm{~F}$ & OS & - & A \\
\hline K.C. & 14 & M & OS & - & A \\
\hline D.K. & 17 & $\mathrm{~F}$ & OS & - & $\mathrm{E}$ \\
\hline M.M.M. & 16 & $\mathrm{~F}$ & OS & - & E \\
\hline M.K. & 14 & M & OS & + & A \\
\hline O.F.K & 10 & M & OS & - & A \\
\hline B.G. & 13 & M & OS & - & $\mathrm{E}$ \\
\hline H.N.K. & 14 & $\mathrm{~F}$ & OS & - & A \\
\hline A.T. & 11 & $\mathrm{~F}$ & OS & - & A \\
\hline E.S & 12 & $\mathrm{~F}$ & ES & - & A \\
\hline B.S. & 13 & $\mathrm{~F}$ & ES & - & A \\
\hline S.O. & 14 & M & ES & - & A \\
\hline M.Y. & 15 & $\mathrm{~F}$ & ES & - & A \\
\hline G.K. & 13 & $\mathrm{~F}$ & ES & + & A \\
\hline D.K. & 7 & $\mathrm{~F}$ & ES & - & E \\
\hline A.I.C. & 11 & $\mathrm{M}$ & ES & - & A \\
\hline
\end{tabular}

Abbreviations: A, alive; E, exitus; ES, Ewing sarcoma; F, female; M, male; OS, osteosarcoma. 


\section{Results}

The preoperative and postoperative PET SUVmax values of the primary and metastasis regions were compared in both surviving patients and those that died. The primary region median SUVmax value decreased from 7.35 to 2.5 with treatment in the surviving patients $(n=16)$ while there was no significant decrease in the primary region median PET SUVmax value in the deceased patients $(n=4)$ $(\mathrm{P}<0.001)$ (Table 2). Enhancement in the metastasis area on PET was seen in 10 bone tumor patients. The pre- and post-treatment median PET SUVmax values in the metas- tasis region of these 10 patients decreased from 2.1 to 0 (involvement disappeared) in the surviving patients ( $\mathrm{n}=$ 7) but only from 4.8 to 3.2 in the deceased patients ( $n=$ 3) $(\mathrm{P}<0.01)$ (Table 3$)$. Evaluation of survival results showed that $28.6 \%$ of the patients receiving only classical treatment died while all patients in the group where sorafenib and anti-VEGF had been added had survived $(\mathrm{P}=0.006)$ (Figure 3). This study included only 20 cases for comparison of combination therapy benefits so we admit this as the limitation of our study.

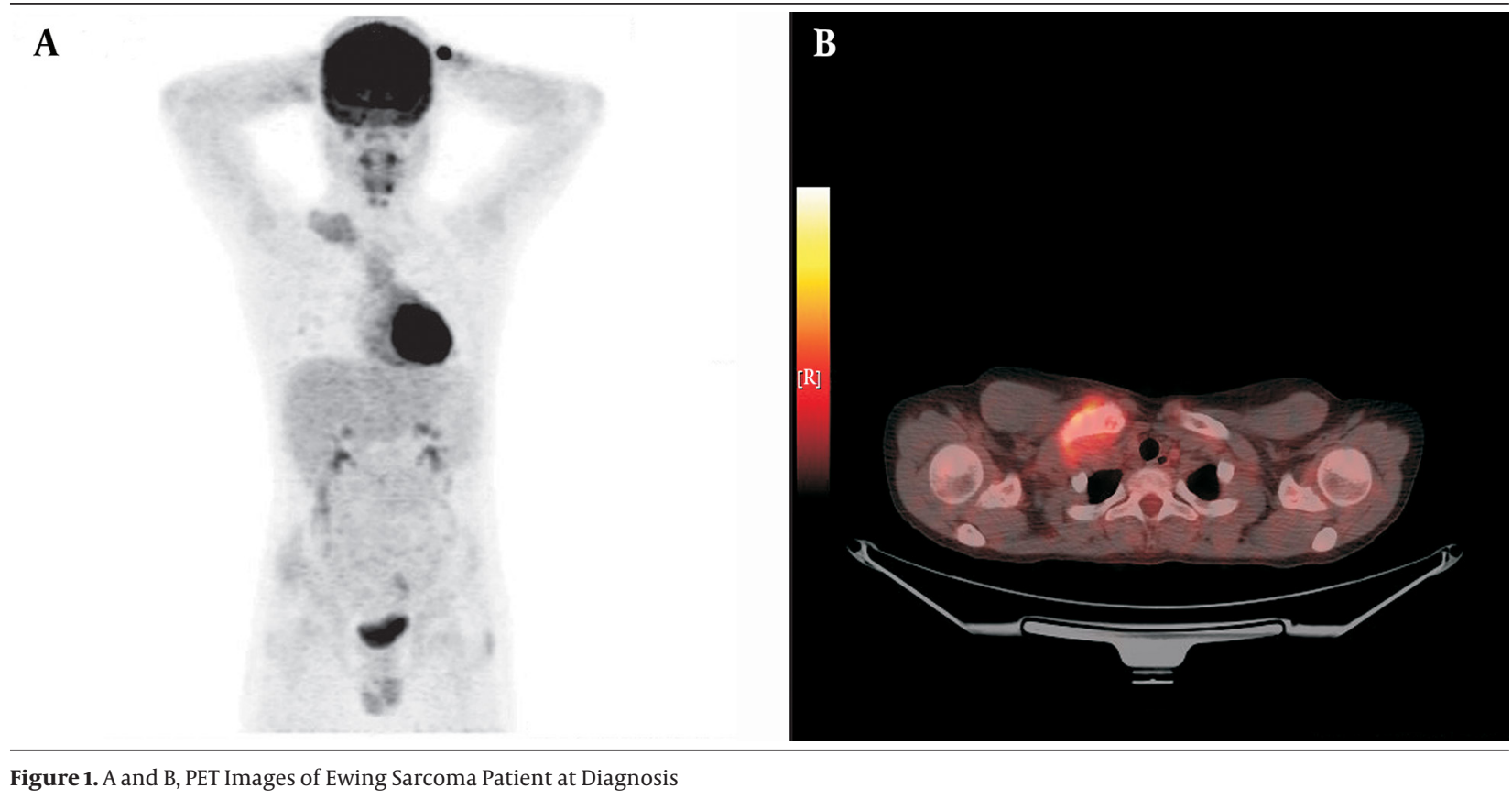

Figure 1. A and B, PET Images of Ewing Sarcoma Patient at Diagnosi

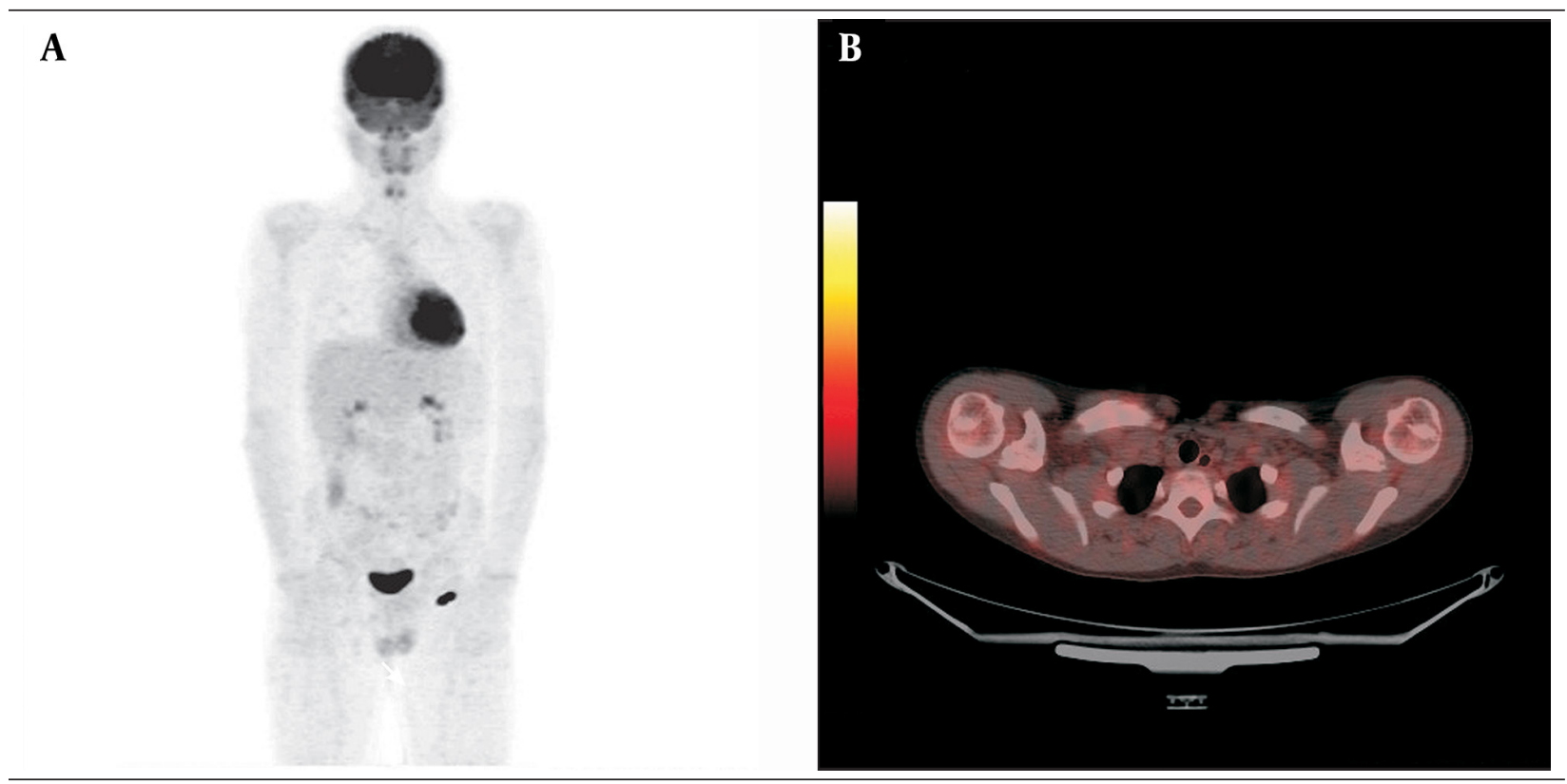

Figure 2. A and B, PET Images of Ewing Sarcoma Patient After Combination Treatment 
Tacyildiz N et al.

\begin{tabular}{|c|c|c|c|}
\hline Patient (n) & Psuvmax1 Inferior-Superior Limit (Median) & PSUVmax2 Inferior-Superior Limit (Median) & P Value \\
\hline Alive (16) & $3.2-25.5(7.35)$ & $0-6.6(2.5)$ & 0.001 \\
\hline Exitus (4) & $8.7-24.8(14.1)$ & $2.3-24(11.3)$ & 0.001 \\
\hline
\end{tabular}

${ }^{a}$ Descriptions: PSUVmax1, primary region before treatment SUVmax value; PSUVmax2, primary region after treatment SUVmax value.

\begin{tabular}{lccc}
\hline \multicolumn{2}{l}{ Table 3. Metastasis Region's PET SUV Max Value Before Treatment and After Treatment ${ }^{\mathrm{a}}$} & & \\
\hline Patient (n) & MSUVmax1 Inferior-Superior Limit (Median) & MSUVmax2 Inferior-Superior Limit (Median) & P Value \\
\hline Alive (7) & $2.1-4(2.1)$ & 0 & 0.01 \\
Exitus (3) & $3-9.4(4.8)$ & $3-5(3.2)$ & 0.01 \\
\hline
\end{tabular}

a Descriptions: PSUVmax1, primary region before treatment SUVmax value; PSUVmax2, primary region after treatment SUVmax value.

Figure 3. The Positive Effect of Sorafenib and Anti-VEGF Combination on Survival

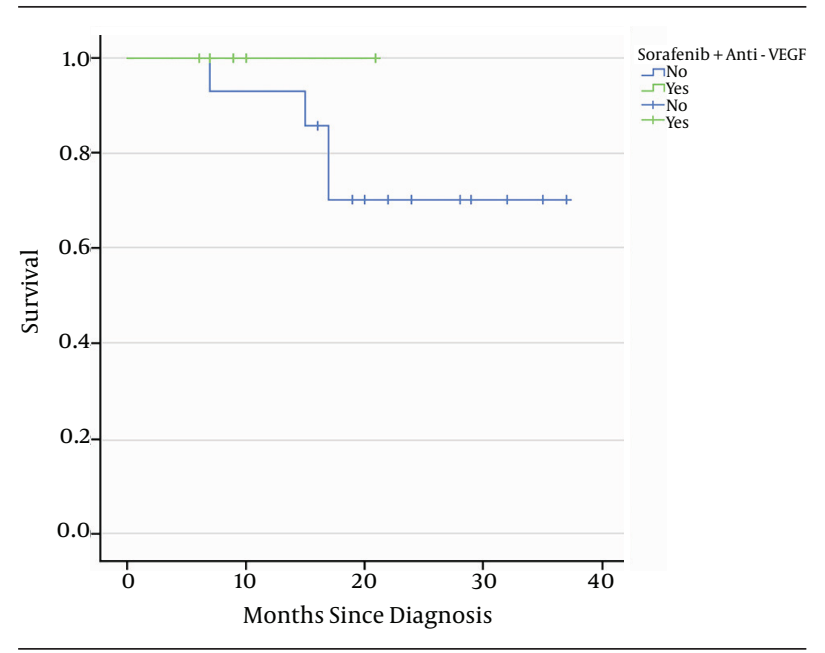

All patients added sorafenib and anti-VEGF are alive $(\mathrm{P}=0.006)$.

\section{Discussion}

The treatment success rate is low in metastatic bone tumors. However, we would like to stress that it is possible to take some prognostic risk factors into account and use non-standard treatments to increase the treatment success rate in patients who have metastatic disease at the time of diagnosis or a low surgical necrosis rate. We would therefore like to discuss the positive effect of the sorafenib and anti-VEGF combination on survival and also the use of PET as an ancillary imaging method for determining the treatment response in childhood bone tumors as in many other cancers.

The use of the oral multikinase inhibitor sorafenib with the targeted treatment agent anti-VEGF monoclonal antibody potentiates the effect of both agents. This combination increases treatment success with a significant improvement in survival rates in bone tumors as in many other cancers (9).

The treatment success has improved in osteosarcoma with the use of multiagent chemotherapy and surgical resection in recent years. The disease-free survival rate in localized resectable disease is now $60-70 \%$. However, new treatment agents are still needed to increase the success rate in metastatic disease (10-12). Grignani et al. explored activity of sorafenib in relaps and unresectable osteosarcoma patients as a second or third-line treatment agent (13). Maki et al. performed a multicenter phase II study and showed that sorafenib had activity aganist sarcoma patients as a single agent (14). Besides, sorafenib was associated with antitumor activity in advanced soft tissue sarcoma patients. In our opinion, sorafenib as a first line targeted therapy, shows antitumor activity and promises in osteosarcoma patients but still deserves further investigation.

Ewing sarcoma patients present with metastasis in approximately $25 \%$ of cases (15). The use of stem cell transplantation following myeloablative chemotherapy and addition of new targeted treatment agents can be considered in addition to systemic chemotherapy and radiotherapy in these cases $(16,17)$. We found that the addition of the sorafenib and anti-VEGF combination to classical treatment in our clinic led to survival of all bone tumor patients while $28.6 \%$ of those receiving classical treatment died. This significant contribution to survival makes it necessary to emphasize the sorafenib and anti-VEGF combination in childhood metastatic bone tumors. This combination was especially reported in adult solid tumors, not in childhood bone tumors. Our first favorable results may encourage clinicians to treat metastatic bone tumors despite the small number of patients included in the study.

A positive correlation between 18F-FDG enhancement on PET and disease aggressiveness, histopathological grading and staging is reported for childhood bone tumors. Many studies have found PET results to be quite sensitive and reliable in determining the treatment response (18-20). Quartuccio et al. compared the diagnostic performance of PET and conventional imaging for staging and follow up of pediatric osteosarcoma and skeletal Ewing sarcoma. They emphasized that PET provides diagnostic benefit in bone tumors (21). We similarly found the PET method to be very valuable in evaluating disease spread in childhood bone tumors at the time of diagnosis, preoperatively following neoadjuvant treatment, and 
Tacyildiz N et al.

postoperatively. The method was able to provide objective results (tumor necrosis rate) to evaluate treatment response as in histopathological evaluation. Certainly prospective studies are needed to define the best imaging algorithm in the follow up of patients with pediatric bone sarcoma. We also found that patients where sorafenib and anti-VEGF were added to treatment showed decreased involvement of the primary and metastasis regions on PET and that the method was sensitive in demonstrating this response. The fact that the metastasis region was controlled as much as the primary region and regarding survival rate during follow-up increased the importance of PET. It is obvious that the sorafenib and anti-VEFG combination makes it more possible to control the pathological involvement of the metastasis regions.

In conclusion, 18F-PET seems to be a reliable method to determine the spread of the disease and to evaluate the response before and after neoadjuvant treatment in patients with metastatic bone sarcoma. The addition of sorafenib and Anti-VEGF to the treatment has improved the PET response and similarly the survival duration of the patients.

\section{Footnote}

Authors' Contribution:Study concept and design: Nurdan Tacyildiz and Hikmet Gulsah Tanyildiz; acquisition of data: Hikmet Gulsah Tanyildiz; analysis and interpretation of data: Hikmet Gulsah Tanyildiz and Handan Ugur Dincaslan; drafting of the manuscript: Nurdan Tacyildiz, Hikmet Gulsah Tanyildiz, Cigdem Soydal; critical revision of the manuscript for important intellectual content: Nurdan Tacyildiz, Elgin Ozkan, Hikmet Gulsah Tanyildiz, Ozlem Kucuk, and Gulsan Yavuz; statistical analysis: Yusuf Yildiz, Nurdan Tacyildiz, and Emel Unal; administrative, technical, and material support: Yusuf Yildiz, Ozlem Kucuk, Cigdem Soydal, and Gulsan Yavuz; study supervision: Nurdan Tacyildiz, Hikmet Gulsah Tanyildiz, Gulsan Yavuz, and Emel Unal.

\section{References}

1. Hillner BE, Siegel BA, Shields AF, Liu D, Gareen IF, Hunt E, et al. Relationship between cancer type and impact of PET and PET/ CT on intended management: findings of the national oncologic PET registry. J Nucl Med. 2008;49(12):1928-35. doi: 10.2967| jnumed.108.056713. [PubMed:18997054]

2. Dimitrakopoulou-Strauss A, Strauss LG, Heichel T, Wu H, Burger C, Bernd L, et al. The role of quantitative (18)F-FDG PET studies for the differentiation of malignant and benign bone lesions. J $\mathrm{Nucl}$ Med. 2002;43(4):510-8. [PubMed: 11937595]

3. Charest M, Hickeson M, Lisbona R, Novales-Diaz JA, Derbekyan V, Turcotte RE. FDG PET/CT imaging in primary osseous and soft tissue sarcomas: a retrospective review of 212 cases. Eur J Nucl Med Mol Imaging. 2009;36(12):1944-51. doi: 10.1007/s00259-009-12030. [PubMed:19593561]

4. Fletcher JW, Djulbegovic B, Soares HP, Siegel BA, Lowe VJ, Lyman GH, et al. Recommendations on the use of 18F-FDG PET in oncology. J Nucl Med. 2008;49(3):480-508. doi: 10.2967| jnumed.107.047787. [PubMed: 18287273]

5. Lan XL, Zhang YX, Wu ZJ, Jia Q, Wei H, Gao ZR. The value of dual time point (18)F-FDG PET imaging for the differentiation between malignant and benign lesions. Clin Radiol. 2008;63(7):756-
64. doi: 10.1016/j.crad.2008.01.003. [PubMed: 18555033]

6. Muheremu A, Niu X. Positron emission tomography/computed tomography for bone tumors (Review). Oncol Lett. 2015;9(2):5226. doi:10.3892/ol.2014.2728. [PubMed: 25621021]

7. Glade Bender JL, Adamson PC, Reid JM, Xu L, Baruchel S, Shaked Y, et al. Phase I trial and pharmacokinetic study of bevacizumab in pediatric patients with refractory solid tumors: a Children's Oncology Group Study. J Clin Oncol. 2008;26(3):399-405. doi: 10.1200/JCO.2007.11.9230. [PubMed:18202416]

8. Widemann BC, Kim A, Fox E, Baruchel S, Adamson PC, Ingle AM, et al. A phase I trial and pharmacokinetic study of sorafenib in children with refractory solid tumors or leukemias: a Children's Oncology Group Phase I Consortium report. Clin Cancer Res. 2012;18(21):601122. doi:10.1158/1078-0432.CCR-11-3284. [PubMed: 22962440]

9. Navid F, Baker SD, McCarville MB, Stewart CF, Billups CA, Wu J, et al. Phase I and clinical pharmacology study of bevacizumab, sorafenib, and low-dose cyclophosphamide in children and young adults with refractory/recurrent solid tumors. Clin CancerRes. 2013;19(1):236-46. doi:10.1158/1078-0432.CCR-12-1897. [PubMed: 23143218]

10. Meyers PA, Schwartz CL, Krailo M, Kleinerman ES, Betcher D, Bernstein ML, et al. Osteosarcoma: a randomized, prospective trial of the addition of ifosfamide and/or muramyl tripeptide to cisplatin, doxorubicin, and high-dose methotrexate. J Clin Oncol. 2005;23(9):200411. doi:10.1200/JCO.2005.06.031. [PubMed:15774791]

11. Shaheen M, Deheshi BM, Riad S, Werier J, Holt GE, Ferguson PC, et al. Prognosis of radiation-induced bone sarcoma is similar to primary osteosarcoma. Clin Orthop Relat Res. 2006;450:76-81. doi: 10.1097/01.blo.0000229315.58878.c1. [PubMed: 16906097]

12. Meyers PA, Schwartz CL, Krailo MD, Healey JH, Bernstein ML, Betcher D, et al. Osteosarcoma: the addition of muramyl tripeptide to chemotherapy improves overall survival--a report from the Children's Oncology Group. J Clin Oncol. 2008;26(4):633-8. doi:10.1200/JCO.2008.14.0095. [PubMed: 18235123]

13. Grignani G, Palmerini E, Dileo P, Asaftei SD, D'Ambrosio L, Pignochino Y, et al. A phase II trial of sorafenib in relapsed and unresectable high-grade osteosarcoma after failure of standard multimodal therapy: an Italian Sarcoma Group study. Ann Oncol. 2012;23(2):508-16.doi:10.1093/annonc/mdr151. [PubMed:21527590]

14. Maki RG, D'Adamo DR, Keohan ML, Saulle M, Schuetze SM, Undevia SD, et al. Phase II study of sorafenib in patients with metastatic or recurrent sarcomas. J Clin Oncol. 2009;27(19):3133-40. doi:10.1200/JCO.2008.20.4495. [PubMed: 19451436]

15. Cotterill SJ, Ahrens S, Paulussen M, Jurgens HF, Voute PA, Gadner H, et al. Prognostic factors in Ewing's tumor of bone: analysis of 975 patients from the European Intergroup Cooperative Ewing's Sarcoma Study Group. JClin Oncol. 2000;18(17):3108-14. [PubMed:10963639]

16. Wagner LM, Crews KR, Iacono LC, Houghton PJ, Fuller CE, McCarville $\mathrm{MB}$, et al. Phase I trial of temozolomide and protracted irinotecan in pediatric patients with refractory solid tumors. Clin Cancer Res. 2004;10(3):840-8. [PubMed: 14871959]

17. Wagner LM, McAllister N, Goldsby RE, Rausen AR, McNall-Knapp RY, McCarville MB, et al. Temozolomide and intravenous irinotecan for treatment of advanced Ewing sarcoma. Pediatr Blood Cancer. 2007;48(2):132-9. doi:10.1002/pbc.20697. [PubMed:16317751]

18. Lakkaraju A, Patel CN, Bradley KM, Scarsbrook AF. PET/CT in primary musculoskeletal tumours: a step forward.EurRadiol.2010;20(12):295972. doi:10.1007/s00330-010-1862-z. [PubMed: 20577880]

19. Treglia G, Salsano M, Stefanelli A, Mattoli MV, Giordano A, Bonomo L. Diagnostic accuracy of (1)(8)F-FDG-PET and PET/CT in patients with Ewing sarcoma family tumours: a systematic review and a meta-analysis. Skeletal Radiol. 2012;41(3):249-56. doi: 10.1007/s00256-011-1298-9. [PubMed: 22072239]

20. Caldarella C, Salsano M, Isgro MA, Treglia G. The Role of Fluorine-18-fluorodeoxyglucose Positron Emission Tomography in Assessing the Response to Neoadjuvant Treatment in Patients with Osteosarcoma. Int J Mol Imaging. 2012;2012:870301. doi: 10.1155/2012/870301. [PubMed: 23008768]

21. Quartuccio N, Fox J, Kuk D, Wexler LH, Baldari S, Cistaro A, et al. Pediatric bone sarcoma: diagnostic performance of (1)(8)F-FDG PET/CT versus conventional imaging for initial staging and follow-up. AJR Am J Roentgenol. 2015;204(1):153-60. doi: 10.2214/ AJR.14.12932. [PubMed: 25539251] 